PROCEEDINGS OF THE

AMERICAN MATHEMATICAL SOCIETY

Volume 138, Number 3, March 2010, Pages 777-786

S 0002-9939(09)10129-6

Article electronically published on October 20, 2009

\title{
DOMINANCE OF A RATIONAL MAP TO THE COBLE QUARTIC
}

\author{
SUKMOON HUH \\ (Communicated by Ted Chinburg)
}

\begin{abstract}
We show the dominance of the restriction map from a moduli space of stable sheaves on the projective plane to the Coble sixfold quartic. With the dominance and the interpretation of a stable sheaf on the plane in terms of hyperplane arrangements, we expect these tools to reveal the geometry of the Coble quartic.
\end{abstract}

\section{INTRODUCTION}

Let $C$ be a smooth non-hyperelliptic curve of genus 3 over complex numbers. Then $C$ is embedded into $\mathbb{P}_{2} \simeq \mathbb{P} H^{0}\left(K_{C}\right)^{*}$ by canonical embedding as a plane quartic curve. The moduli space $S U_{C}\left(2, K_{C}\right)$ of semistable vector bundles of rank 2 with canonical determinant over $C$ is known to be a hypersurface in $\mathbb{P}_{7}$, called the 'Coble quartic', 3], 13. Let $\mathcal{W}^{r}$ be the closure of the following set

$$
\left\{E \in S U_{C}\left(2, K_{C}\right) \mid h^{0}(C, E) \geq r+1\right\} .
$$

Then we have the following inclusions [14 on the Brill-Noether loci,

$$
S U_{C}\left(2, K_{C}\right) \supset \mathcal{W} \supset \mathcal{W}^{1} \supset \mathcal{W}^{2} \supset \mathcal{W}^{3}=\emptyset
$$

where $\mathcal{W}=\mathcal{W}^{0}$. Many properties on the geometry of these Brill-Noether loci have been discovered in 14 .

Let $\bar{M}\left(c_{1}, c_{2}\right)$ be the moduli space of stable sheaves of rank 2 with the Chern classes $\left(c_{1}, c_{2}\right)$ on the projective plane. The dimension of this space is known to be $4 c_{2}-3$ if $c_{1}=0$ [2] and $4 c_{2}-4$ if $c_{1}=-1$ [9]. Then there exists a rational map [8]

$$
\Phi_{k}: \bar{M}(1, k) \rightarrow S U_{C}\left(2, K_{C}\right), 1 \leq k \leq 4,
$$

defined by sending $E$ to $\left.E\right|_{C}$. It is shown in [8] that $\Phi_{k}$ is a dominant map to $\mathcal{W}^{2}, \mathcal{W}^{1}$ and $\mathcal{W}$, for $k=1,2,3$, respectively. In this article, we give a proof of the dominance of the rational map $\Phi_{4}$. This is equivalent to the dominance of the rational map from $\bar{M}(3,6)$ to $S U_{C}\left(2,3 K_{C}\right)$ by twisting. For a general bundle $E \in S U_{C}\left(2,3 K_{C}\right)$, we embed $C$ with $\mathbb{P}_{2}$ into a Grassmannian $\operatorname{Gr}(5,2)$ and take the pull-back of the universal quotient bundle of $G r(5,2)$ to $\mathbb{P}_{2}$. This bundle is shown to be stable and have the Chern classes $(3,6)$.

Received by the editors January 15, 2008, and, in revised form, April 26, 2009.

2000 Mathematics Subject Classification. Primary 14D20; Secondary 14M15.

This article is part of the revised version of the author's thesis at the University of Michigan. The author would like to express his deepest gratitude to his advisor, Professor Igor Dolgachev. The author is also grateful to the referee for many suggestions.

(C)2009 American Mathematical Society Reverts to public domain 28 years from publication 
As a quick consequence, we can obtain the old result that $S U_{C}\left(2, K_{C}\right)$ is unirational since $\bar{M}(1,4)$ is rational. The unirationality implies the rationally connectedness. We see how we can obtain a rational curve through two general points of the Coble quartic in terms of hyperplane arrangements.

The restriction of vector bundles on $\mathbb{P}_{2}$ to plane curves was also studied in [7, where the author investigated the restriction of the tangent bundle of $\mathbb{P}_{2}$ to plane curves and gave the conditions for a vector bundle $E$ on a plane curve to be a pull-back of the tangent bundle of $\mathbb{P}_{2}$, twisted by $\mathcal{O}_{\mathbb{P}_{1}}(-1)$.

For the background on vector bundles, we suggest [12] as a good reference.

\section{Embedding Plane QUARTiCs in Grassmannians}

Let $E$ be a semistable vector bundle of rank 2 with the determinant $3 K_{C}$ over $C$, i.e. $E \in S U_{C}\left(2,3 K_{C}\right)$. By the following lemma, we can obtain a morphism

$$
\varphi: C \rightarrow G r\left(H^{0}(E), 2\right)
$$

sending $p \in C$ to the 2-dimensional quotient space $E_{p}$ of $H^{0}(E)$.

Lemma 2.1. $H^{1}(C, E)=0$ and $E$ is globally generated.

Proof. $H^{1}(E) \simeq H^{0}\left(E^{*} \otimes K_{C}\right) \neq 0$ implies the existence of a nonzero homomorphism $E \rightarrow \mathcal{O}_{C}\left(K_{C}\right)$ which contradicts the semistability of $E$. Now, by the same argument, we have $H^{1}(E(-p))=0$ for all $p \in C$. From the long exact sequence of the sequence

$$
0 \rightarrow E(-p) \rightarrow E \rightarrow E_{p} \rightarrow 0,
$$

we obtain the surjective evaluation map $H^{0}(E) \rightarrow E_{p}$, which implies the global generation of $E$.

In fact, the morphism $\varphi$ fits in the diagram

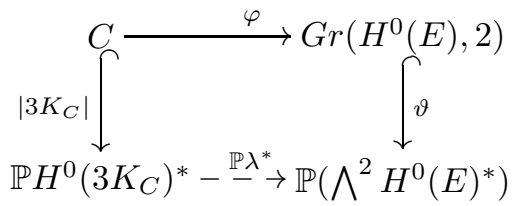

where $\vartheta$ is the Plucker embedding and $\mathbb{P} \lambda^{*}$ comes from the dual of the homomorphism

$$
\lambda: \bigwedge^{2} H^{0}(E) \rightarrow H^{0}\left(\bigwedge^{2} E\right) \simeq H^{0}\left(3 K_{C}\right) .
$$

By the following lemma, $\mathbb{P} \lambda^{*}$ is an embedding and so is $\varphi$ for general $E$.

Lemma 2.2. The homomorphism $\lambda$ is surjective for general $E \in S U_{C}\left(2,3 K_{C}\right)$.

Proof. If $E$ is stable, then by the Nagata-Severi theorem 11, we have the exact sequence for $E\left(-K_{C}\right)$,

$$
0 \rightarrow \mathcal{O}(D) \rightarrow E\left(-K_{C}\right) \rightarrow \mathcal{O}\left(K_{C}-D\right) \rightarrow 0,
$$

where $D$ is a divisor of degree 1 . For general $E$, we have $H^{0}\left(E\left(-K_{C}\right)\right)=0$, i.e. we can assume that $H^{0}(\mathcal{O}(D))=0$; i.e. $D$ is non-effective.

Let $L=\mathcal{O}\left(K_{C}+D\right)$ and $F=\mathcal{O}\left(2 K_{C}-D\right)$. Then we have

$$
0 \rightarrow L \rightarrow E \rightarrow F \rightarrow 0 \text {. }
$$


Note that $h^{0}(L)=3, h^{0}(F)=5$ and $h^{1}(L)=h^{1}(F)=0$ and, from the long exact sequence of the above sequence, we have

$$
H^{0}(E) \simeq H^{0}(L) \oplus H^{0}(F),
$$

and hence it is enough to show the surjectivity of the map

$$
H^{0}(L) \otimes H^{0}(F) \rightarrow H^{0}(L \otimes F) \simeq H^{0}\left(3 K_{C}\right) .
$$

For every $p \in C, h^{0}(L(-p))=2+h^{1}(L(-p))=2+h^{0}(p-D)=2$ since $D$ is not effective. Hence, we can have a map from $C$ to $\operatorname{Gr}\left(2, H^{0}(L)\right)$ sending $p$ to $H^{0}(L(-p))$. Since $\operatorname{Gr}\left(2, H^{0}(L)\right) \simeq \mathbb{P}_{2}$, we can choose $W \in \operatorname{Gr}\left(2, H^{0}(L)\right)$ which is not the same as $H^{0}(L(-p))$ for any $p \in C$. Then by the choice of $W$, it does not have base locus on $C$. Now consider the map

$$
W \otimes H^{0}(F) \rightarrow H^{0}\left(3 K_{C}\right) .
$$

By the Base-Point-Free Pencil Trick [1, the kernel of this map is isomorphic to $H^{0}\left(C, F \otimes L^{-1}\right)$, and this is isomorphic to $H^{0}\left(K_{C}-2 D\right)$. Note that $h^{0}\left(K_{C}-2 D\right)=h^{0}(2 D)$ by the Riemann-Roch theorem. If $h^{0}(2 D)=0$, then $W \otimes H^{0}(F)$ is isomorphic to $H^{0}\left(3 K_{C}\right)$ by the counting of the dimensions. Hence, it is enough to show that $H^{0}(2 D)=0$ for general $E$.

Assume that $h^{0}(2 D)>0$, and then $\mathcal{O}(2 D)$ is an element of the theta divisor in $\operatorname{Pic}^{2}(C)$. The map

$$
\operatorname{Pic}^{1}(C) \rightarrow \operatorname{Pic}^{2}(C),
$$

defined by $D \mapsto 2 D$, is a finite surjective map of degree 64 . Hence the subvariety of $\operatorname{Pic}^{1}(C)$ whose elements are $D$ such that $h^{0}(D)=0$ and $h^{0}(2 D)>0$ is of 2 dimensions. For these divisors $D$, the extensions of $\mathcal{O}\left(K_{C}-D\right)$ by $\mathcal{O}(D)$ are parametrized by $\mathbb{P}_{3}$, which means that the vector bundles that do not satisfy $h^{0}(2 D)=0$ are of at most 5 dimensions. Hence $h^{0}(2 D)=0$ in general.

Now, for the 5-dimensional subspace $V \subset H^{0}(E)$, we have the following diagram:

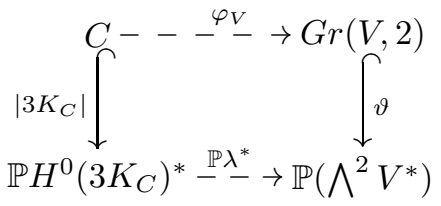

Consider a natural map

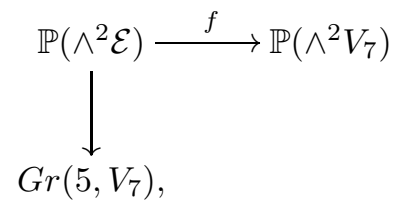

where $\mathcal{E}$ is the universal subbundle, $V_{7}$ is a 7-dimensional vector space and $\operatorname{Gr}\left(5, V_{7}\right)$ is the Grassmannian of 5-dimensional subspaces of $V_{7}$. Over $\left[V_{5}\right] \in G r\left(5, V_{7}\right)$, the fibre $\wedge^{2} V_{5}$ is linearly embedded into $\wedge^{2} V_{7}$.

Lemma 2.3. The image of $f$ is the secant variety of $G r\left(2, V_{7}\right) \subset \mathbb{P}\left(\wedge^{2} V_{7}\right)$, and its dimension is equal to 17. 
Proof. Let $[x] \in \operatorname{Im}(f)$; i.e. there exists a $V_{5}$ such that $x \in \wedge^{2} V_{5}$. Consider $G=$ $\operatorname{Gr}\left(2, V_{5}\right) \subset \mathbb{P}\left(\wedge^{2} V_{5}\right)$. Since the secant variety of $G$ is $\mathbb{P}\left(\wedge^{2} V_{5}\right)$, we can express $x$ by

$$
(v \wedge w) \text { or }\left(v_{1} \wedge v_{2}+v_{3} \wedge v_{4}\right),
$$

which proves that $\operatorname{Im}(f)$ is contained in the secant variety of $\operatorname{Gr}\left(2, V_{7}\right)$.

Now we show the inclusion $\operatorname{Sec}\left(\operatorname{Gr}\left(2, V_{7}\right)\right) \hookrightarrow \operatorname{Im}(f)$. Assume that $x$ is a general point in the secant variety. This means that

$$
x=v_{1} \wedge v_{2}+v_{3} \wedge v_{4},
$$

where $U=\left\langle v_{1}, v_{2}, v_{3}, v_{4}\right\rangle$ is a 4 -dimensional space. For any $V_{5} \supset U$, we have $x \in \wedge^{2} V_{5}$. This shows that

$$
\operatorname{Sec}\left(G r\left(2, V_{7}\right)\right)=\operatorname{Im}(f),
$$

since both sides are closed subvarieties of $\mathbb{P}\left(\wedge^{2} V_{7}\right)$. Also the set of such $V_{5}$ is 2-dimensional and $\operatorname{dim} f^{-1}([x])=2$. Hence the dimension of $\operatorname{Im}(f)$ is 17 , since $\operatorname{dim}\left(\mathbb{P}\left(\wedge^{2} \mathcal{E}\right)\right)=19$.

Remark 2.4. $\operatorname{Gr}\left(2, V_{7}\right)$ is a Scorza variety of defect $\delta=4$ [16]. So, it is known that $\operatorname{dim} \operatorname{Sec}\left(\operatorname{Gr}\left(2, V_{7}\right)\right)=17$.

Lemma 2.5. For general $E \in S U_{C}\left(2,3 K_{C}\right)$ and general 5-dimensional vector subspace $V \subset H^{0}(E)$, the restriction of $\lambda$ to $\wedge^{2} V$,

$$
\lambda: \bigwedge^{2} V \rightarrow H^{0}\left(3 K_{C}\right),
$$

is an isomorphism.

Proof. In the proof of (2.2), let

$$
V_{7}:=W \oplus V_{5},
$$

where $V_{5} \simeq H^{0}(F)$. In fact, we can take any $V_{5} \subset V_{7}$ with $V_{5} \cap H^{0}(L)=0$. Then, the restriction of $\lambda$ to $\wedge^{2} V_{7}$ is also surjective. Let $K=\operatorname{ker}(\lambda)$ be the 11-dimensional subspace of $\wedge^{2} V_{7}$. Consider an incidence variety $\mathcal{R} \subset G r\left(5, V_{7}\right) \times \mathbb{P}(K)$,

$$
\mathcal{R}=\left\{\left(V_{5},[x]\right) \mid x \in \wedge^{2} V_{5} \cap K\right\} .
$$

We have the following diagram:

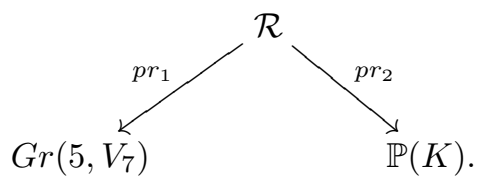

It is enough to show that the map $p r_{1}$ is not dominant, which means that for the general $V_{5} \subset V_{7}$ not in the image of $p r_{1}$, we have the surjection in the assertion. Assume that $p r_{1}$ is dominant, then

$$
\operatorname{dim}(\mathcal{R}) \geq 10 .
$$

If we consider again the map

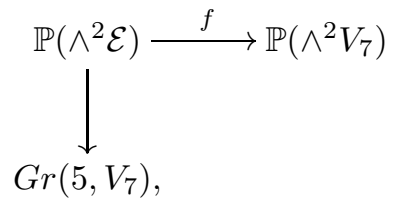


then the image of $p r_{2}$ in $\mathbb{P}(K)$ is the intersection of $\operatorname{Im}(f)=\operatorname{Sec}\left(G r\left(2, V_{7}\right)\right)$ with $\mathbb{P}(K)$ in $\mathbb{P}\left(\wedge^{2} V_{7}\right) \simeq \mathbb{P}_{20}$. Since $\operatorname{Im}(f)$ is 17 -dimensional, we have

$$
7 \leq \operatorname{dim} \operatorname{Im}\left(p r_{2}\right) \leq 10 .
$$

It is clear that $\mathbb{P}(K)$ contains a point in $\operatorname{Sec}\left(G r\left(2, V_{7}\right)\right)$, but not in $\operatorname{Gr}\left(2, V_{7}\right)$. The fibre over this point in $\mathcal{R}$ is isomorphic to $G r(1,3) \simeq \mathbb{P}_{2}$. Thus the dimension of $\operatorname{Im}\left(p r_{2}\right)$ is greater than 7 .

Now assume that $\operatorname{dim} \mathbb{P}(K) \cap \operatorname{Sec}(G r(2,7)) \geq 8$. In the proof of (2.2), we have

$$
K \cap\left(W \wedge V_{5}\right)=(0),
$$

if $V_{5} \cap W=(0)$. If $V_{5} \cap W \neq(0)$, the intersection is always $\left[\wedge^{2} W\right]$. Let us consider the canonical map

$$
s: W \otimes V_{7} / W \rightarrow \wedge^{2} V_{7} / \wedge^{2} W .
$$

For all $V_{5}$ with $V_{5} \cap W=(0)$, the images in $\wedge^{2} V_{7} / \wedge^{2} W$ are the same as a 10dimensional vector space. If we take the preimage of this space in $\wedge^{2} V_{7}$, then it is the union of $W \wedge V_{5}$ for all $V_{5}$, which is now an 11-dimensional space. Note that $K \cap\left(W \wedge V_{5}\right)=\left[\wedge^{2} W\right]$ if $W \cap V_{5} \neq(0)$. Let us denote by $D$ the projectivization of the preimage of $s\left(W \otimes V_{7} / W\right)$ in $\wedge^{2} V_{7}$. Then $D$ is a 10-dimensional subvariety of $\mathbb{P}\left(\wedge^{2} V_{7}\right)$ and it intersects with $\mathbb{P}(K)$ at the unique point $\left[\wedge^{2} W\right]$. In fact, $D$ is the projective tangent space $\mathbb{P} T_{[W]} G r\left(2, V_{7}\right)$ of $G r\left(2, V_{7}\right)$ at $[W]$ in $\mathbb{P}\left(\wedge^{2} V_{7}\right)$. Recall that

$$
\begin{aligned}
& T_{[W]} G r\left(2, V_{7}\right)=\operatorname{Hom}\left(W, V_{7} / W\right) \simeq W^{*} \otimes V_{7} / W \\
& T_{\left[\wedge^{2} W\right]} \mathbb{P}\left(\wedge^{2} V_{7}\right)=\operatorname{Hom}\left(\wedge^{2} W, \wedge^{2} V_{7} / \wedge^{2} W\right) .
\end{aligned}
$$

The differential map of the Plücker embedding at $[W]$ is defined as follows: $x=$ $w^{*} \otimes e \in T_{[W]} G r\left(2, V_{7}\right)$ is sent to the map

$$
w_{1} \wedge w_{2} \mapsto s\left(\left(w^{*}\left(w_{1}\right) w_{2}-w_{1} w^{*}\left(w_{2}\right)\right) \otimes e\right),
$$

where $W=\left\langle w_{1}, w_{2}\right\rangle$. This explains the assertion.

Now since the union of the secant lines of $G r\left(2, V_{7}\right)$ passing through $\left[\wedge^{2} W\right]$ is 11-dimensional and $\mathbb{P}(K) \cap \operatorname{Sec}\left(\operatorname{Gr}\left(2, V_{7}\right)\right)$ is of dimension $\geq 8$, we can pick an element $[U] \in \mathbb{P}(K) \cap G r\left(2, V_{7}\right)$, and then the secant line $\overline{[U][W]}$ lies in $\mathbb{P}(K)$. From the condition on $W, U$ and $W$ span a 4-dimensional subspace of $V_{7}$. In particular, general points on the secant line $\overline{[U][W]}$ are indecomposable. Let $p$ be such a point. Since $\operatorname{Sing}\left(\operatorname{Sec}\left(\operatorname{Gr}\left(2, V_{7}\right)\right)\right)=\operatorname{Gr}\left(2, V_{7}\right)$ [16], the dimension of $T_{p}\left(\operatorname{Sec}\left(G r\left(2, V_{7}\right)\right)\right)$ is 17. Note that

$$
T_{p}\left(\operatorname{Sec}\left(G r\left(2, V_{7}\right)\right)\right)=\left\langle T_{[W]} G, T_{[U]} G\right\rangle .
$$

Since

$$
T_{p}\left(\mathbb{P}(K) \cap \operatorname{Sec}\left(G r\left(2, V_{7}\right)\right)\right)=\mathbb{P}(K) \cap T_{p}\left(\operatorname{Sec}\left(G r\left(2, V_{7}\right)\right)\right)
$$

is at least 8-dimensional, $\mathbb{P}(K)$ intersects $T_{[W]} G$ along at least 1-dimensional subspace, which is a contradiction because $\mathbb{P}(K) \cap D$ is a single point. 
From the previous lemma, we have the commutative diagram

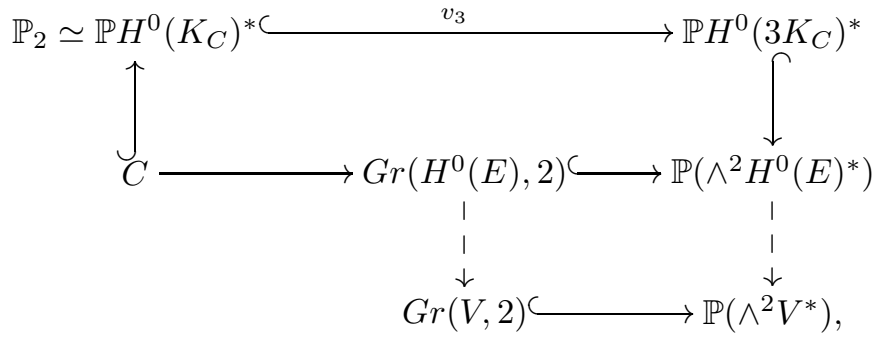

where the composite of the two vertical maps on the right,

$$
\mathbb{P} H^{0}\left(3 K_{C}\right)^{*} \hookrightarrow \mathbb{P}\left(\wedge^{2} H^{0}(E)^{*}\right) \rightarrow \mathbb{P}\left(\wedge^{2} V^{*}\right),
$$

is an isomorphism and $v_{3}$ is the 3-tuple Veronese embedding; i.e. $v_{3}$ is given by the complete linear system $\left|\mathcal{O}_{\mathbb{P}_{2}}(3)\right|$. In particular, $C$ is embedded into $\operatorname{Gr}(V, 2)$. Note that $C$ is non-degenerate in $\mathbb{P}_{9} \simeq \mathbb{P}\left(\wedge^{2} V^{*}\right)$ due to the Riemann-Roch theorem and the Noether theorem.

Corollary 2.6. General element $E$ in $S U_{C}\left(2,3 K_{C}\right)$ is generated by a 5-dimensional subspace of $H^{0}(E)$.

\section{Embedding the Projective Plane into Grassmannian}

In the diagram (10), the projective plane $\mathbb{P} H^{0}\left(K_{C}\right)^{*} \simeq \mathbb{P}_{2}$ is embedded into the projective space $\mathbb{P}\left(\wedge^{2} V^{*}\right) \simeq \mathbb{P}_{9}$ by the Plücker embedding.

Lemma 3.1. For general $E \in S U_{C}\left(2,3 K_{C}\right)$, there exists a 5-dimensional vector subspace $V \subset H^{0}(E)$ such that $\mathbb{P} H^{0}\left(K_{C}\right)^{*}$ is embedded into $G r(V, 2)$ in the diagram (10).

Proof. Let $V \subset H^{0}(E)$ be a 5-dimensional subspace selected in 2.5 and assume that $\mathbb{P} H^{0}\left(K_{C}\right)^{*}$ is not embedded into $\operatorname{Gr}(V, 2)$. Recall that $G r(V, 2)$ is cut out by the 4-dimensional projectively linear family of quadrics of rank 6 in $\mathbb{P}_{9}$ whose singular locus is $\mathbb{P}_{3}$ contained in $G r(V, 2)$ as the Schubert variety of lines through a point corresponding to the quadric in $\mathbb{P}_{4}[15$. Let $Q(p)$ be one of the quadrics of rank 6 containing $\operatorname{Gr}(V, 2)$ which does not contain $S$, where $p$ is a point in $\mathbb{P}_{4}$ and $S$ is the image of $\mathbb{P}_{2}$ by $v_{3}$. Since $v_{3}^{-1}(Q(p))$ is a plane sextic curve, we have

$$
v_{3}^{-1}(Q(p))=C+C^{\prime},
$$

where $C^{\prime}$ is a conic. First, assume that $\operatorname{Gr}(V, 2) \cap S=C+C^{\prime}$. If we consider the incidence variety $Z_{C}=\{(l, x) \mid x \in l\} \subset C \times \mathbb{P}_{4}$, we have a diagram

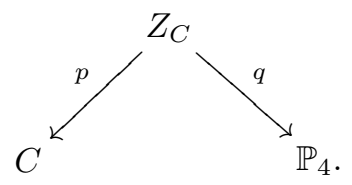

Let $S_{C}$ be the image of $q$ in $\mathbb{P}_{4}$. If $S_{C}$ is degenerate, i.e. there exists a hyperplane $\mathbb{P}_{3} \subset \mathbb{P}_{4}$ containing $S_{C}$, then $C$ is contained in some Grassmannian $\operatorname{Gr}(4,2) \subset$ $G r(V, 2)$ and, in particular, $C$ is contained in $\mathbb{P}_{5}$, the Plücker space of $G r(4,2)$, 
which is a contradiction to the non-degeneracy of $C$ in $\mathbb{P}_{9}$. Similarly we can define $Z_{C^{\prime}}$ and $S_{C^{\prime}}$. Recall the well known fact that

$$
\operatorname{deg}(C)=\operatorname{deg}\left(S_{C}\right) \cdot \operatorname{deg}(q) .
$$

If $\operatorname{deg}\left(S_{C}\right)=1$, i.e. $S_{C}$ is a plane in $\mathbb{P}_{4}$, then $C$ must be contained in $\mathbb{P}_{3}(p)$, the singular locus of a quadric $Q(p)$ for $p \in S_{C}$, which is a contradiction to the fact that $C \subset \mathbb{P}_{9}$ is nondegenerate. Hence $\operatorname{deg}\left(S_{C}\right) \geq 2$ and $\operatorname{so} \operatorname{deg}(q) \leq 6$. This implies that the number of points in $\mathbb{P}_{3}(p) \cap C$ is less than 7 for $p \in S_{C}$. Since the intersection of $S_{C}$ and $S_{C^{\prime}}$ is at most 1-dimensional in $S_{C}$, we still have 2-dimensional choices for $p$ for which $\mathbb{P}_{3}(p) \cap\left(C+C^{\prime}\right)=\mathbb{P}_{3}(p) \cap C$ is less than 7 points. We can also have the same conclusion on the intersection number of $\mathbb{P}_{3}(p) \cap\left(C+C^{\prime}\right)$ in the case when $\operatorname{Gr}(V, 2) \cap S$ is the proper subset of $C+C^{\prime}$ since it still contains $C$. Now choose $p \in \mathbb{P}_{4}$ such that the singular locus $\mathbb{P}_{3}(p)$ of $Q(p)$ meets $C+C^{\prime}$ with $k$ points where $0<k<7$. We have the commutative diagram

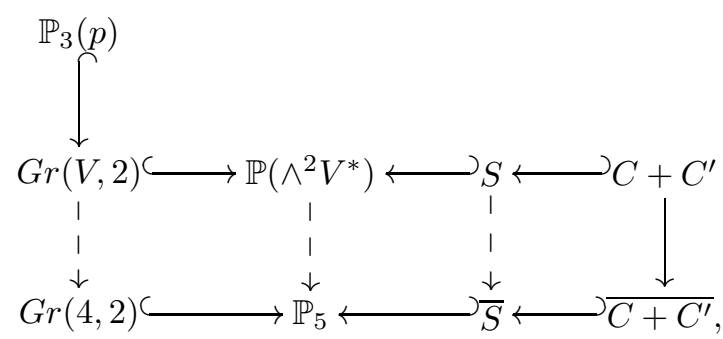

where $\bar{S}, \overline{C+C^{\prime}}$ are the images of $S, C+C^{\prime}$, respectively, via the projection, and the image of $G r(V, 2)$ lies in the image of the quadric $Q$, i.e. the Grassmannian $\operatorname{Gr}(4,2) \subset \mathbb{P}_{5}$. Let $Q^{\prime}$ be another quadric cutting $\operatorname{Gr}(V, 2)$ with singular locus $\mathbb{P}_{3}^{\prime}$. Since $\mathbb{P}_{3} \cap \mathbb{P}_{3}^{\prime}$ is a single point, the image of $Q^{\prime}$ by the projection is $\mathbb{P}_{5}$. Thus the image of $\operatorname{Gr}(V, 2)$ is $\operatorname{Gr}(4,2)$. Note that the degree of $\overline{C+C^{\prime}}$ is $18-k$ and the degree of $\bar{S}$ is $9-k$ since $\mathbb{P}_{3}(p) \cap S=\mathbb{P}_{3}(p) \cap\left(C+C^{\prime}\right)$. If $Q(p)$ contains $S$ for all such $p \in S_{C}$, then all quadrics containing $\operatorname{Gr}(V, 2)$ of rank 6 should contain $S$ since $S_{C}$ is nondegenerate in $\mathbb{P}_{4}$. In particular, $G r(V, 2)$ should contain $S$, which is against the assumption. So there exists a $p \in S_{C}$ for which $S$ is not contained in $Q(p)$. Thus the image of $S$ by the projection is also not contained in the image of $Q(p)$, i.e. $G r(4,2)$. But the degree of intersection $G r(4,2) \cap \bar{S}$ is $2 \times(9-k)<18-k$, which is a contradiction to the fact that this intersection contains $\overline{C+C^{\prime}}$.

Let $U_{V}$ and $\overline{U_{V}}$ be the universal subbundle and quotient bundle of $\operatorname{Gr}(V, 2)$, respectively. With the condition on $V$ in the previous lemma, let

$$
E_{V}:=v_{3}^{*} \overline{U_{V}}
$$

which implies that the restriction of $E_{V}$ to $C$ is $E$, i.e. $\left.E_{V}\right|_{C}=E$.

Lemma 3.2. $E_{V}$ is stable with the Chern classes $(3,6)$, i.e. $E_{V} \in \bar{M}(3,6)$.

Proof. Since the first Chern class of $\overline{U_{V}}$ is the hyperplane section of $\operatorname{Gr}(V, 2)$ in $\mathbb{P}\left(\wedge^{2} V^{*}\right)$ and $v_{3}$ is the 3 -tuple Veronese embedding, we get $c_{1}\left(E_{V}\right)=3$.

By the choice of $V$, we have an exact sequence

$$
0 \rightarrow G \rightarrow V \otimes \mathcal{O}_{\mathbb{P}_{2}} \rightarrow E_{V} \rightarrow 0,
$$

where $G$ is the kernel of the surjection $V \otimes \mathcal{O}_{\mathbb{P}_{2}} \rightarrow E_{V}$ and $V$ is a 5-dimensional vector subspace of $H^{0}\left(E_{V}\right)$. In particular, $h^{0}\left(E_{V}\right) \geq 5$. By the choice of $E$, we 
have $h^{0}\left(\left.E_{V}(-1)\right|_{C}\right)=0$. From the long exact sequence of cohomology of the exact sequence

$$
\left.0 \rightarrow E_{V}(-5) \rightarrow E_{V}(-1) \rightarrow E_{V}(-1)\right|_{C} \rightarrow 0,
$$

we have

$$
H^{0}\left(E_{V}(-5)\right) \simeq H^{0}\left(E_{V}(-1)\right) .
$$

For a line $H \subset \mathbb{P}_{2},\left.E_{V}\right|_{H} \simeq \mathcal{O}_{H}(a) \oplus \mathcal{O}_{H}(3-a)$ for $a=2$ or 3 since $E_{V}$ is globally generated. In particular, $h^{0}\left(\left.E_{V}(-k)\right|_{H}\right)=0$ for $k \geq 4$. From the long exact sequence of cohomology of the exact sequence

$$
\left.0 \rightarrow E_{V}(-k-1) \rightarrow E_{V}(-k) \rightarrow E_{V}(-k)\right|_{H} \rightarrow 0,
$$

we have $h^{0}\left(E_{V}(-k-1)\right)=h^{0}\left(E_{V}(-k)\right)$ for all $k \geq 4$. Since $h^{0}\left(E_{V}(-k)\right)=0$ for sufficiently large $k$, we have $h^{0}\left(E_{V}(-k)\right)=0$ for $k \geq 4$ and in particular, $h^{0}\left(E_{V}(-1)\right)=h^{0}\left(E_{V}(-5)\right)=0$; i.e. $h^{0}\left(E_{V}(-k)\right)=0$ for all $k \geq 1$. Hence the vector bundle $E_{V}$ is stable.

Again, let $H$ be a line in $\mathbb{P}_{2}$. From the exact sequence

$$
\left.0 \rightarrow E_{V}(-1) \rightarrow E_{V} \rightarrow E_{V}\right|_{H} \rightarrow 0,
$$

we get $h^{0}\left(E_{V}\right) \leq h^{0}\left(\left.E_{V}\right|_{H}\right)$. Since $\left.E_{V}\right|_{H} \simeq \mathcal{O}_{H}(a) \oplus \mathcal{O}_{H}(3-a)$ for $a=2$ or 3 , $h^{0}\left(\left.E_{V}\right|_{H}\right)=5$ and so $h^{0}\left(E_{V}\right) \leq 5$. Thus we obtain $h^{0}\left(E_{V}\right)=\operatorname{dim} V=5$.

Now from the long exact sequence of cohomology of (13), we have $h^{0}\left(\mathbb{P}_{2}, G\right)=0$. If we twist (13) by -1 , we have $h^{1}\left(\mathbb{P}_{2}, G(-1)\right)=0$. For any line $l \subset \mathbb{P}_{2}$, consider the exact sequence

$$
\left.0 \rightarrow G(-1) \rightarrow G \rightarrow G\right|_{l} \rightarrow 0 .
$$

From the above statement, we get $H^{0}\left(\left.G\right|_{l}\right)=0$. Since $c_{1}(G)=-c_{1}\left(E_{V}\right)=-3$, we have $\left.G\right|_{l} \simeq \mathcal{O}_{l}(a) \oplus \mathcal{O}_{l}(b) \oplus \mathcal{O}_{l}(c)$ with $a+b+c=-3$. The only choice from the vanishing of $H^{0}\left(\left.G\right|_{l}\right)$ is $(a, b, c)=(-1,-1,-1)$. Hence $G$ is a uniform vector bundle of rank 3 on $\mathbb{P}_{2}$ with the splitting type $(-1,-1,-1)$. From the classification of such bundles [5], we have

$$
G \simeq \mathcal{O}_{\mathbb{P}_{2}}(-1)^{\oplus 3}
$$

In particular, $c_{2}(G)=3$ and so $c_{2}\left(E_{V}\right)=6$.

Since we can pick an element $E_{V} \in \bar{M}(3,6)$ mapping to a general element $E \in$ $S U_{C}\left(2,3 K_{C}\right)$, the rational map

$$
\bar{M}(3,6) \rightarrow S U_{C}\left(2,3 K_{C}\right)
$$

is dominant. By twisting the map (14) with $\mathcal{O}_{\mathbb{P}_{2}}(-1)$ and $\mathcal{O}_{C}\left(-K_{C}\right)$, we have the following main theorem.

Theorem 3.3. The restriction map

$$
\Phi_{4}: \bar{M}(1,4) \rightarrow S U_{C}\left(2, K_{C}\right)
$$

is dominant.

Remark 3.4. Dolgachev and Kapranov 4 showed that the logarithmic bundles $E(\mathcal{H})$ attached to the general hyperplane arrangement $\mathcal{H}=\left(H_{1}, \cdots, H_{6}\right)$ in $\mathbb{P}_{2}$ form an open Zariski subset $U \subset \bar{M}(3,6)$. For these bundles $E(\mathcal{H})$, we have a Steiner resolution

$$
0 \rightarrow \mathcal{O}_{\mathbb{P}_{2}}(-1)^{\oplus 3} \rightarrow \mathcal{O}_{\mathbb{P}_{2}}^{\oplus 5} \rightarrow E(\mathcal{H}) \rightarrow 0 .
$$


From this, we have a 5-dimensional space $V=H^{0}\left(\mathbb{P}_{2}, E(\mathcal{H})\right)$. Tensoring the exact sequence

$$
0 \rightarrow \mathcal{O}_{\mathbb{P}_{2}}(-4) \rightarrow \mathcal{O}_{\mathbb{P}_{2}} \rightarrow \mathcal{O}_{C} \rightarrow 0
$$

by $E(\mathcal{H})$, we can consider $V$ as a subspace of $H^{0}\left(C,\left.E(\mathcal{H})\right|_{C}\right)$, which is 8-dimensional. As we have seen already in the proof of 3.2. the bundle $E_{V}$ has a Steiner resolution, pulled back from the universal exact sequence on the Grassmannian $\operatorname{Gr}(V, 2)$. This motivates the whole argument in this paper.

Since $\bar{M}(1,4)$ is rational and the map $\Phi_{4}$ is dominant, $S U_{C}\left(2, K_{C}\right)$ is unirational. It implies that $S U_{C}\left(2, K_{C}\right)$ is rationally connected and so rationally chainconnected. Let $\mathcal{H}=\left(H_{0}, \cdots, H_{6}\right)$ be a general arrangement of 6 lines on $\mathbb{P}_{2}$ and then we can associate a logarithmic bundle $E(\mathcal{H}) \in \bar{M}(3,6)$ to $\mathcal{H}$. It is known [4] that the logarithmic bundles $E(\mathcal{H})$ form an open Zariski subset of $\bar{M}(3,6)$ and, after twisting by $\mathcal{O}_{\mathbb{P}_{2}}(-1), \bar{M}(1,4)$. Let $\mathcal{F}$ be a family of arrangements of 6 lines on $\mathbb{P}_{2}$ and let $E(\mathcal{F})$ be the closure of the subvariety of $\bar{M}(1,4)$ whose closed points correspond to $E(\mathcal{H}) \otimes \mathcal{O}_{\mathbb{P}_{2}}(-1)$ with $\mathcal{H} \in \mathcal{F}$.

Proposition 3.5. $S U_{C}\left(2, K_{C}\right)$ is rationally chain-connected. In fact, any two general points in $S U_{C}\left(2, K_{C}\right)$ can be connected by at most 6 rational curves which can be described explicitly.

Proof. Let us consider a special type of arrangement of 6 lines. Let $H_{0}, H_{1}, \cdots, H_{5}$ be 6 lines in general position on $\mathbb{P}_{2}$ and let $p$ be a fixed point on $H_{0}$ in general position. If we fix $H_{1}, \cdots, H_{5}$, then we have a 1-dimensional family $\mathcal{F}$ of 6 lines with $H_{0}$ moving. Consider a map

$$
\Psi: \mathbb{P}_{1}(\mathcal{F}) \rightarrow S U_{C}\left(2, K_{C}\right)
$$

sending $\mathcal{H}$ to $\left.E(\mathcal{H})(-1)\right|_{C}$. Since $S U_{C}\left(2, K_{C}\right)$ is projective, this map is a morphism [6]. Clearly $\Psi$ is not a constant map; otherwise $\Phi_{4}$ is also a constant map, which is not true. From the fact that logarithmic bundles associated to 6 lines in general position form an open Zariski subset of $\bar{M}(3,6)$ and $\Phi_{4}$ is dominant, we can find a 1-dimensional family of 6 lines $\mathcal{F}$ which maps to a rational curve on $S U_{C}\left(2, K_{C}\right)$ via $\Psi$ for a general element of $S U_{C}\left(2, K_{C}\right)$. Furthermore, for two general elements $E_{1}, E_{2} \in \bar{M}(3,6)$, we can find 6 families of 6 lines $\mathcal{F}_{i}, 1 \leq i \leq 6$, as above such that the arrangements corresponding to $E_{1}, E_{2}$ lie in $\mathcal{F}_{1}, \mathcal{F}_{6}$ respectively and $\mathcal{F}_{i} \cap \mathcal{F}_{i+1} \neq$ $\emptyset$. From this fact with the dominance of $\Phi_{4}$, we can find 6 rational curves passing through two general points on $S U_{C}\left(2, K_{C}\right)$.

Remark 3.6. Note that we can choose these rational curves not contained in the singular locus of $S U_{C}\left(2, K_{C}\right)$ which is the Kummer variety of $\operatorname{Pic}^{2}(C)$. Let $\widetilde{S}$ be a desingularization by the blow-up [10] and consider the proper transform of the previous 6 rational curves on $S U_{C}\left(2, K_{C}\right)$. It shows the rationally chain-connectedness of $\widetilde{S}$, and since $\widetilde{S}$ is smooth, it implies the rational connectedness; i.e. the chain of these 6 curves can be deformed to a rational curve and its image on $S U_{C}\left(2, K_{C}\right)$ will give us a rational curve through two general points. 


\section{REFERENCES}

1. E. Arbarello, M. Cornalba, P. A. Griffiths, and J. Harris, Geometry of algebraic curves, Vol. I, Grundlehren der Mathematischen Wissenschaften [Fundamental Principles of Mathematical Sciences], vol. 267, Springer-Verlag, New York, 1985. MR770932 (86h:14019)

2. W. Barth, Moduli of vector bundles on the projective plane, Invent. Math. 42 (1977), 63-91. MR0460330 (57:324)

3. Arthur B. Coble, Algebraic geometry and theta functions, revised printing. American Mathematical Society Colloquium Publication, vol. X, American Mathematical Society, Providence, RI, 1961. MR0123958 (23:A1279)

4. I. Dolgachev and M. Kapranov, Arrangements of hyperplanes and vector bundles on $\mathbf{P}^{n}$, Duke Math. J. 71 (1993), no. 3, 633-664. MR.1240599(95e:14029)

5. G. Elencwajg, Les fibrés uniformes de rang 3 sur $\mathbf{P}_{2}(\mathbf{C})$ sont homogènes, Math. Ann. 231 (1977/78), no. 3, 217-227. MR0481133(58:1278)

6. Robin Hartshorne, Algebraic geometry, Graduate Texts in Mathematics, No. 52, SpringerVerlag, New York, 1977. MR 0463157 (57:3116)

7. G. Hein, The tangent bundle of $\mathbf{P}^{2}$ restricted to plane curves, Complex analysis and geometry (Trento, 1995), Pitman Res. Notes Math. Ser., vol. 366, Longman, Harlow, 1997, pp. 137-140. MR:1477446 (98j:14047)

8. S. Huh, Moduli spaces of stable sheaves on the projective plane and on the plane quartic curve, Ph.D. thesis, University of Michigan, May 2007.

9. Klaus Hulek, Stable rank-2 vector bundles on $\mathbf{P}_{2}$ with $c_{1}$ odd, Math. Ann. 242 (1979), no. 3, 241-266. MR545217 (80m:14011)

10. Young-Hoon Kiem, The stringy E-function of the moduli space of rank 2 bundles over a Riemann surface of genus 3, Trans. Amer. Math. Soc. 355 (2003), no. 5, 1843-1856 (electronic). MR $1953528(2003 \mathrm{j}: 14045)$

11. Herbert Lange, Higher secant varieties of curves and the theorem of Nagata on ruled surfaces, Manuscripta Math. 47 (1984), no. 1-3, 263-269. MR7444323 (85f:14043)

12. Shigeru Mukai, An introduction to invariants and moduli, Cambridge Studies in Advanced Mathematics, vol. 81, Cambridge University Press, Cambridge, 2003; translated from the 1998 and 2000 Japanese editions by W. M. Oxbury. MR2004218 (2004g:14002)

13. M. S. Narasimhan and S. Ramanan, $2 \vartheta$-linear systems on abelian varieties, Vector bundles on algebraic varieties (Bombay, 1984), Tata Inst. Fund. Res. Stud. Math., vol. 11, Tata Inst. Fund. Res., Bombay, 1987, pp. 415-427. MR893605 (88j:14014)

14. W. M. Oxbury, C. Pauly, and E. Previato, Subvarieties of $\mathrm{SU}_{C}(2)$ and $2 \vartheta$-divisors in the Jacobian, Trans. Amer. Math. Soc. 350 (1998), no. 9, 3587-3614. MR1467474 (98m:14034)

15. J. G. Semple and L. Roth, Introduction to algebraic geometry, Oxford, at the Clarendon Press, 1949. MR0034048 (11:535d)

16. F. L. Zak, Tangents and secants of algebraic varieties, Translations of Mathematical Monographs, vol. 127, American Mathematical Society, Providence, RI, 1993; translated from the Russian manuscript by the author. MR.1234494 (94i:14053)

Korea Institute for Advanced Study, Hoegiro 87, Dongdaemun-gu, Seoul 130-722, Republic OF KoREA

E-mail address: sukmoonh@kias.re.kr 